\title{
APLICANDO A DECOMPOSIÇÃO EM VALORES SINGULARES NO PROCESSAMENTO DE IMAGENS
}

\author{
APPLYING SINGULAR VALUE DECOMPOSITION (SVD) IN IMAGE \\ PROCESSING
}

\author{
DANILO P. FURTADO \\ CRISTIANE OLIVEIRA DE FARIA ${ }^{\mathrm{b}}$ DIANA SASAKI ${ }^{\mathrm{c}}$
}

\section{Resumo}

A decomposição em valores singulares (SVD) é uma das mais importantes fatorações de matrizes pois tem sua existência garantida e pode ser aplicada a quaisquer tipos de matrizes. Essa versatilidade permitiu que a SVD pudesse ser usada em diversas aplicações, por exemplo na área de processamento de imagens. Neste trabalho, apresentamos um algoritmo simples para o cálculo numérico da decomposição SVD baseado no método das potências e investigamos duas aplicações na área de processamento de imagens: compressão e autenticação de imagens. Os experimentos numéricos realizados têm como objetivo principal demonstrar aplicações práticas e ilustrativas da decomposição SVD e do método das potências, ambos tópicos frequentemente abordados em cursos de Álgebra Linear e Métodos Numéricos nos níveis de graduação e pós-graduação, sendo possíveis de ser reproduzidos e assim motivar o interesse dos alunos nestes temas.

Palavras-chave: Decomposição em valores singulares, Processamento de Imagens, Método das Potências, Compressão de Imagens, Autenticação de Imagens.

\footnotetext{
Abstract

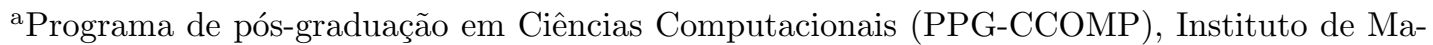
temática e Estatística (IME), Universidade do Estado do Rio de Janeiro (UERJ), RJ, Brasil; ORCID: 0000-0003-4425-2106; E-mail: furtadodp@gmail.com

${ }^{\mathrm{b}}$ Departamento de Análise Matemática, Instituto de Matemática e Estatística - IME, Universidade do Estado do Rio de Janeiro - UERJ, Rio de Janeiro, Brasil; ORCID: 0000-0002-0402-7185; E-mail: cofaria@ime.uerj.br

${ }^{\mathrm{c}}$ Departamento de Matemática Aplicada, Instituto de Matemática e Estatística - IME, Universidade do Estado do Rio de Janeiro - UERJ, Rio de Janeiro, Brasil; ORCID: 0000.0002.0514.9918; E-mail: diana.sasaki@ime.uerj.br
} 
The singular value decomposition (SVD) is one of the most important matrix factorization because it has its existence guaranteed and can be applied to any type of matrix. This versatility made SVD to be used in various applications, such as in image processing. In this work, we present a simple algorithm for numerical calculation of the SVD based on the power method and investigate two applications in image processing: image compression and authentication. The numerical experiments performed intended to demonstrate practical and illustrative applications of the SVD decomposition and the power method, both often presented in Linear Algebra and Numerical Methods courses at the undergraduate and graduate levels, being possible to be reproduced and motivate some interest of the students in these themes.

Keywords: Singular Value Decomposition, Image Processing, Power method, Image Compression, Digital Watermarking.

MSC2010: 15A18, 65F15, 68U10

\section{Introdução}

A decomposição em valores singulares, em inglês Singular Value Decomposition (SVD), é considerada uma das mais importantes fatorizações de matrizes da era computacional pois tem sua existência garantida e pode ser aplicada a quaisquer tipos de matrizes: quadradas, retangulares, singulares e não singulares $[1,2]$. Além disso, soma-se ao fato de que, em 1970, Gene H. Golub juntamente com William Kahan publicaram um algoritmo estável e simples para calcular a decomposição de valor singular difundindo o seu uso em simulações computacionais em várias aplicações e que é usado até hoje [3].

A SVD foi descoberta independentemente por Eugenio Beltrami (1835-1899), por Camille Jordan (1838-1921) e por James Joseph Sylvester (1814-1959), para matrizes quadradas reais. Em 1915, L. Autonne demonstrou a SVD para matrizes quadradas complexas, e em 1936, Carl Eckart e Gale Young consideraram matrizes retangulares gerais [2]. Mais detalhes sobre as contribuições destes matemáticos que são considerados os criadores e desenvolvedores da teoria da SVD podem ser encontrados em [4].

Esta versatilidade em manipular vários tipos de matrizes, quadradas ou nãoquadradas, a possibilidade de redução de dimensionalidade das matrizes permitiu que a decomposição em valores singulares pudesse ser usada em diversas aplicações, tais como: obter aproximações de menor posto para matrizes, calcular pseudoinversas de matrizes não quadradas [1], compressão de imagens [5], autenticação de imagens [6], filtragem de dados sísmicos [7], entre outras. 
Este trabalho está estruturado da seguinte forma: na Seção 2 apresentamos uma breve revisão da teoria da decomposição em valores singulares baseada nas referências [1] e [8]. Na Seção 3, mostramos um algoritmo simples para cálculo numérico da decomposição SVD com base no método das potências e na sua exposição teórica. Em seguida, na Seção 4, são apresentados os resultados da utilização deste algoritmo em duas aplicações na área de processamento de imagens: compressão e autenticação de imagens e finalmente a Conclusão.

Os experimentos numéricos aqui apresentados têm como objetivo principal demonstrar aplicações práticas e ilustrativas da decomposição SVD e do método das potências, ambos tópicos abordados em cursos de Álgebra Linear e Métodos Numéricos nos níveis de graduação e pós-graduação. Dessa forma, o presente trabalho não se propõe a discutir as metodologias e algoritmos mais eficientes encontrados na literatura especializada utilizados nos processos de compressão e autenticação de imagens e sim apresentar exemplos reais que possam ser reproduzidos por alunos dos cursos citados.

\section{A Decomposição em Valores Singulares}

A decomposição em valores singulares é uma fatoração matricial em que uma matriz $A$, pode ser decomposta em três matrizes de acordo com o Teorema 2.1.

Teorema 2.1 (Decomposição em Valores Singulares). Se A for uma matriz $m \times n$, de posto $^{1} k$, então $A$ pode ser expressa como

$$
A=U \Sigma V^{T}
$$

em que $U$ e $V$ são matrizes quadradas ortogonais ${ }^{2}$ de ordem $m$ e $n$, respectivamente, $e \Sigma$ é uma matriz $m \times n$ cujas entradas diagonais são os $k$ valores singulares de $A$ e demais entradas são nulas.

\footnotetext{
${ }^{1}$ A dimensão comum do espaço linha e do espaço coluna de uma matriz $A$ é denominada posto. Para uma matriz $A \in \mathbb{R}^{m, n}$, o posto de $A$ é menor ou igual ao menor valor entre $m$ e $n$ [8].

${ }^{2}$ Uma matriz quadrada $A$ é ortogonal se sua transposta é igual a sua inversa, ou seja, se $A^{-1}=A^{T}$, ou equivalentemente, se $A A^{T}=A^{T} A=I[8]$.
} 
A equação (2.1) pode ser escrita matricialmente na forma:

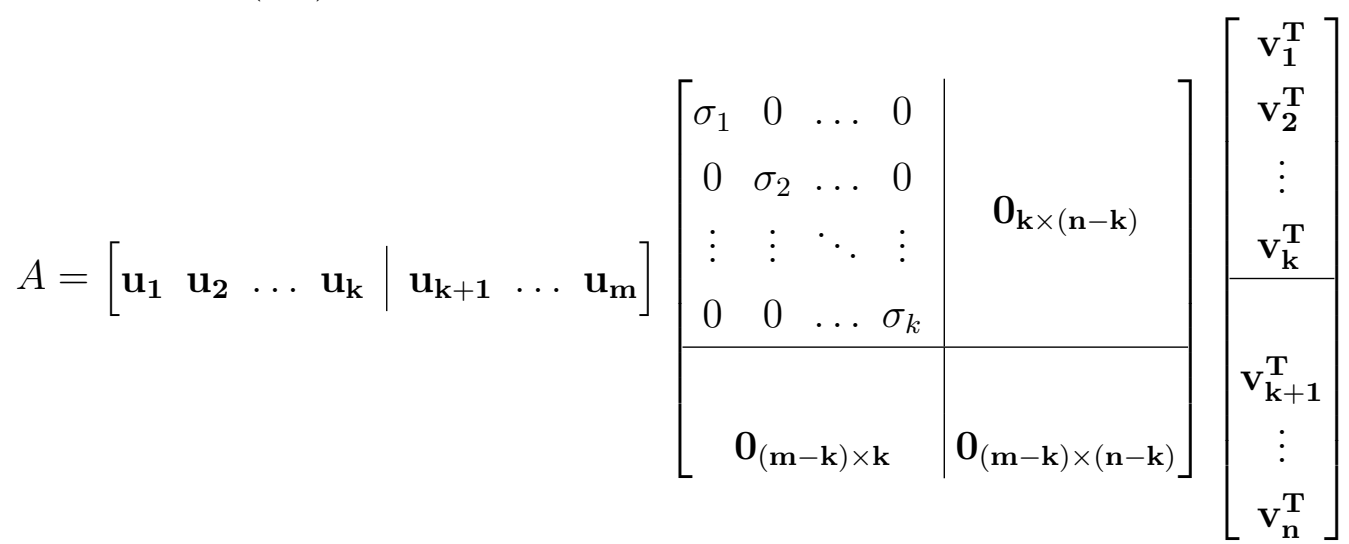

onde $\mathbf{u}_{\mathbf{i}} \in \mathbb{R}^{m}, 1 \leq i \leq m, \sigma_{j} \in \mathbb{R}, 1 \leq j \leq k$, e $\mathbf{v}_{\mathbf{l}}^{\mathbf{T}} \in \mathbb{R}^{n}, 1 \leq l \leq n$. Algebricamente, as linhas e colunas nulas da matriz $\Sigma$ na equação (2.2) são dispensáveis e podem ser eliminadas, pois ao se efetuar o produto das matrizes, os produtos que envolvem blocos nulos como fatores são anulados. Sendo assim, podemos decompor $A$ como:

$$
A=\left[\begin{array}{llll}
\mathbf{u}_{\mathbf{1}} & \mathbf{u}_{\mathbf{2}} & \ldots & \mathbf{u}_{\mathbf{k}}
\end{array}\right]\left[\begin{array}{cccc}
\sigma_{1} & 0 & \ldots & 0 \\
0 & \sigma_{2} & \ldots & 0 \\
\vdots & \vdots & \ddots & \vdots \\
0 & 0 & \ldots & \sigma_{k}
\end{array}\right]\left[\begin{array}{c}
\mathbf{v}_{\mathbf{1}}^{\mathbf{T}} \\
\mathbf{v}_{\mathbf{2}}^{\mathbf{T}} \\
\vdots \\
\mathbf{v}_{\mathbf{k}}^{\mathbf{T}}
\end{array}\right]
$$

que é denominada Decomposição em Valores Singulares Reduzida de $A$. Podemos também escrever a matriz $A$ como:

$$
A=\sigma_{1} \mathbf{u}_{\mathbf{1}} \mathbf{v}_{\mathbf{1}}^{\mathbf{T}}+\sigma_{\mathbf{2}} \mathbf{u}_{\mathbf{2}} \mathbf{v}_{\mathbf{2}}^{\mathbf{T}}+\ldots+\sigma_{\mathbf{k}} \mathbf{u}_{\mathbf{k}} \mathbf{v}_{\mathbf{k}}^{\mathbf{T}}
$$

que é denominada expansão em valores singulares reduzida de $A$. Os vetores $\mathbf{u}_{\mathbf{i}}$ e $\mathbf{v}_{\mathbf{i}}^{\mathbf{T}}$ são denominados vetores singulares à esquerda e à direita de $A$, respectivamente.

A decomposição SVD satisfaz ainda as seguintes propriedades:

P1. $V=\left[\mathbf{v}_{\mathbf{1}} \mathbf{v}_{\mathbf{2}} \ldots \mathbf{v}_{\mathbf{n}}\right]$ diagonaliza ortogonalmente $A^{T} A$.

P2. As entradas diagonais não nulas de $\Sigma$ são $\sigma_{1}=\sqrt{\lambda_{1}}, \sigma_{2}=\sqrt{\lambda_{2}}, \ldots, \sigma_{k}=\sqrt{\lambda_{k}}$, sendo $\lambda_{1}, \lambda_{2}, \ldots, \lambda_{k}$ os autovalores não nulos de $A^{T} A$ associados aos vetores coluna de $V$.

P3. Os vetores colunas de $V$ são ordenados de tal modo que $\lambda_{1} \geq \lambda_{2} \geq \ldots \geq \lambda_{k}>0$.

P4. $\mathbf{u}_{\mathbf{i}}=\frac{\mathbf{A v}_{\mathbf{i}}}{\sigma_{\mathbf{i}}}$.

As demonstrações dessas propriedades, bem como do Teorema 2.1 podem ser encontradas em [8]. 


\section{Algoritmos para o cálculo da decomposição SVD}

A decomposição SVD de uma matriz pode ser obtida, numericamente, por diversos algoritmos tais como: algoritmo de Jacobi, algoritmo de Hestenes, Golub-Kahan, tridiagonalização e iteração QR simétrica, tridiagonalização e divisão e conquista, entre outros [9]. Neste trabalho, apresentamos um algoritmo simples com base no método das potências. Vale ressaltar que a escolha desse algoritmo foi baseada tanto em sua facilidade de implementação quanto em sua presença nas ementas de cursos de graduação e pós-graduação em disciplinas como Álgebra Linear e Métodos Numéricos. Portanto, não se buscou aqui o método mais eficiente para cálculo da decomposição SVD e sim, um método que pudesse ser reproduzido facilmente por alunos destes cursos. Comparações entre vários algoritmos disponíveis para este fim podem ser encontrados em $[9,10]$.

O método das potências é um método iterativo utilizado para obtenção do autovalor dominante de uma matriz e seu correspondente autovetor. Atribui-se uma aproximação inicial arbitrária para o autovetor correspondente ao autovalor dominante que é sucessivamente melhorada até que a precisão requerida seja alcançada. A convergência para o autovalor dominante é simultaneamente obtida [11].

A convergência do método é garantida pelo Teorema 3.1, cuja prova pode ser encontrada em [12, p. 202-203].

Teorema 3.1. Seja A uma matriz simétrica de ordem $n$ com um autovalor dominante $\lambda$ positivo. Se $\mathbf{x}_{\mathbf{0}}$ for um vetor unitário de $\mathbb{R}^{n}$ que não é ortogonal ao autoespaço associado a $\lambda$, então a sequência de potências normalizada ${ }^{3}$

$$
\mathbf{x}_{0}, \mathbf{x}_{1}=\frac{A \mathrm{x}_{\mathbf{0}}}{\left\|A \mathrm{x}_{\mathbf{0}}\right\|}, \mathrm{x}_{\mathbf{2}}=\frac{A \mathrm{x}_{1}}{\left\|A \mathrm{x}_{\mathbf{1}}\right\|}, \ldots, \mathrm{x}_{\mathbf{i}}=\frac{A \mathrm{x}_{\mathbf{i}-\mathbf{1}}}{\left\|A \mathrm{x}_{\mathbf{i}-\mathbf{1}}\right\|}
$$

converge a um autovetor dominante unitário e a sequência

$$
A \mathrm{x}_{1} \cdot \mathbf{x}_{1}, A \mathrm{x}_{2} \cdot \mathbf{x}_{2}, \ldots, A \mathbf{x}_{\mathbf{i}} \cdot \mathbf{x}_{\mathbf{i}}
$$

converge ao autovalor dominante $\lambda$.

A velocidade de convergência do método das potências depende da razão $\frac{\left|\lambda_{2}\right|}{\left|\lambda_{1}\right|}$, onde $\lambda_{1}$ é o autovalor dominante e $\lambda_{2}$ é o segundo autovalor. Portanto, quanto maior for $\left|\lambda_{1}\right|$ quando comparado com $\left|\lambda_{2}\right|$, mais rápida será a convergência [12].

Utilizando as propriedades da decomposição SVD, podemos então aplicar o método das potências à matriz simétrica $A^{T} A$ para calcular o maior autovalor e

\footnotetext{
${ }^{3}$ Neste texto, usamos o termo norma no sentido de norma euclidiana.
} 
seu autovetor associado e, a partir destes, obter o primeiro valor singular $\sigma_{1}$ e os vetores $\mathbf{v}_{\mathbf{1}}$ e $\mathbf{u}_{\mathbf{1}}$. Usando a expansão em valores singulares reduzida Eq. (2.4), podemos escrever:

$$
A^{\prime}=A-\sigma_{1} \mathbf{u}_{\mathbf{1}} \mathbf{v}_{\mathbf{1}}^{\mathbf{T}}=\sigma_{\mathbf{2}} \mathbf{u}_{\mathbf{2}} \mathbf{v}_{\mathbf{2}}^{\mathbf{T}}+\ldots+\sigma_{\mathbf{k}} \mathbf{u}_{\mathbf{k}} \mathbf{v}_{\mathbf{k}}^{\mathbf{T}}
$$

e, portanto, podemos aplicar o método novamente para a matriz $A^{\prime T} A^{\prime}$ e obter o segundo valor singular, e assim sucessivamente, até obtermos todos os valores singulares e vetores associados.

O algoritmo da decomposição SVD utilizando o método das potências, baseado em [8] (Algoritmo 1), é mostrado abaixo em pseudo-código.

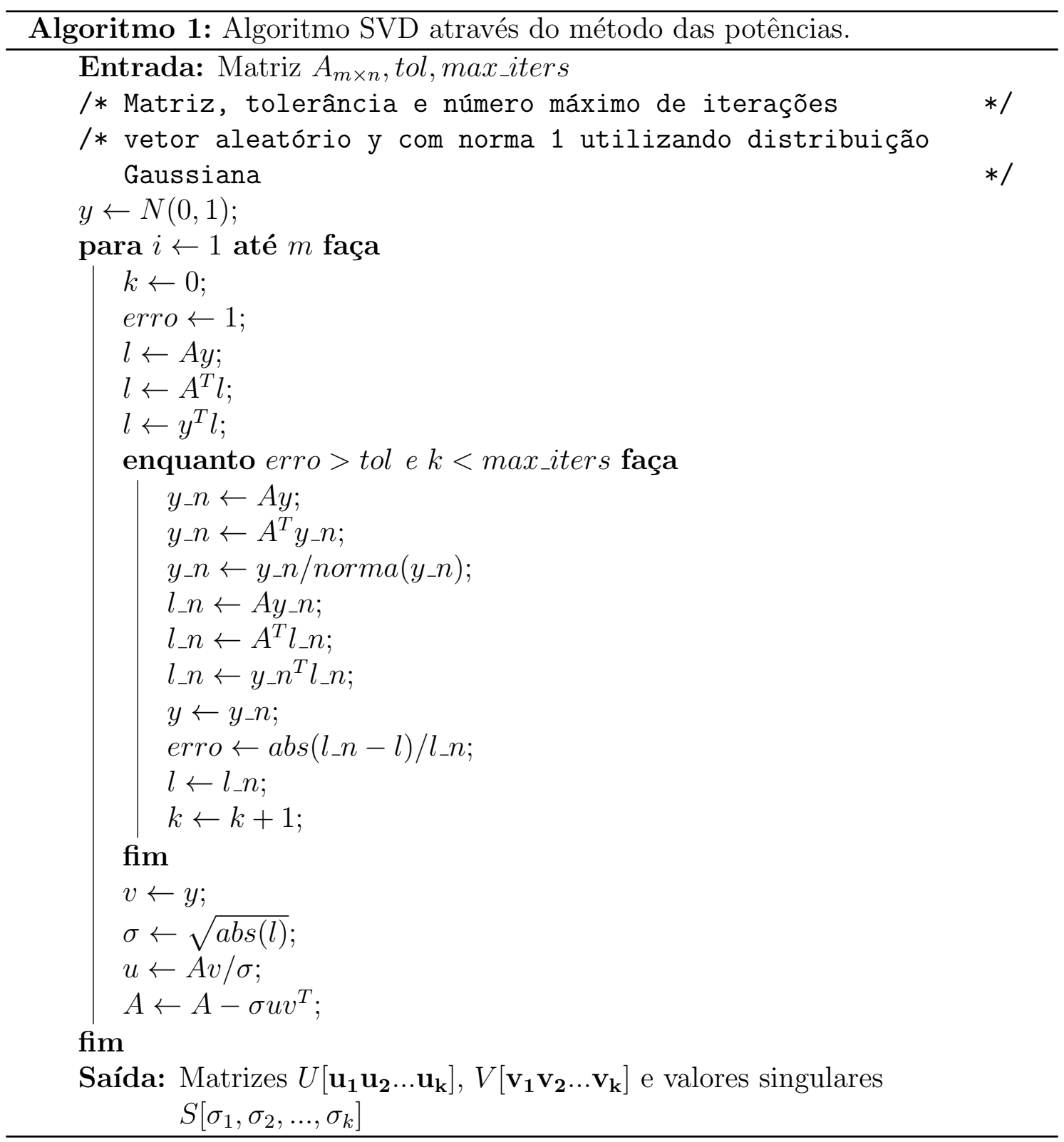




\section{Aplicações ao Processamento de Imagens}

Nesta seção, será mostrada a aplicação da teoria e do algoritmo, descritos nas seções anteriores, em dois tópicos distintos da área de processamento de imagens: compressão e autenticação de imagens. Todos os resultados foram obtidos através de scripts escritos em linguagem Python (3.6.4), utilizando o algoritmo apresentado na seção anterior, em uma workstation portátil com processador intel core i7 (4940MX CPU, 3,10GHz) e 32GB de memória RAM.

\subsection{Compressão de Imagens}

Um dos exemplos de aplicação da decomposição em valores singulares é a Compressão de imagens. Dada uma imagem, que pode ser uma fotografia (imagem natural) ou uma ressonância magnética (imagem médica) deseja-se reduzir ou comprimir a quantidade de informação necessária para sua visualização que deve ser armazenada e/ou transmitida. As imagens naturais são exemplos simples de compressibilidade inerente devido ao alto nível de redundância encontrada nesse tipo de imagem. O termo imagem natural inclui aquelas imagens que estão usualmente presentes no ambiente em que vivemos. Esta definição engloba uma classe geral de imagens que inclui cenas e objetos presentes na natureza, bem como objetos criados pelo homem [13].

Uma imagem em escala cinza pode ser pensada como uma matriz de valores reais $X \in \mathbb{R}^{n \times m}$, onde $n$ e $m$ são os números de pixels na vertical e horizontal, respectivamente.

As Figuras 1(a) e 1(b) mostram exemplos de uma imagem natural e uma imagem gerada por um ruído gaussiano, isto é, onde cada pixel é representado por um valor aleatório seguindo uma distribuição gaussiana ${ }^{4}$, respectivamente. Pode-se verificar o nível de redundância presente em uma imagem, por exemplo, através de um gráfico de dispersão, onde cada ponto possui como coordenadas o valor de um determinado pixel $\left(\mathbf{x}_{1}\right)$ e o valor do pixel vizinho à sua direita $\left(\mathbf{x}_{2}\right)$.

Observa-se claramente nos gráficos mostrados nas Figuras 1(c) e 1(d), que os pixels vizinhos da imagem natural apresentam alto coeficiente de correlação ${ }^{5}(\rho=$

\footnotetext{
${ }^{4} \mathrm{~A}$ distribuição Gaussiana é uma distribuição de probabilidade dada por $p(x)=$ $\frac{1}{\sigma \sqrt{2 \pi}} e^{-\frac{1}{2}\left(\frac{x-\mu}{\sigma}\right)^{2}}$, onde $\mu$ é a média e $\sigma$ é o desvio padrão da distribuição [14].

${ }^{5} \mathrm{O}$ coeficiente de correlação, também conhecido como coeficiente de correlação de Pearson, mede o grau de dependência linear entre duas variáveis através da expressão $\rho(x, y)=$ $\frac{\sum_{i=1}^{n}\left(x_{i}-\bar{x}\right)\left(y_{i}-\bar{y}\right)}{\sqrt{\sum_{i=1}^{n}\left(x_{i}-\bar{x}\right)^{2} \cdot \sum_{i=1}^{n}\left(y_{i}-\bar{y}\right)^{2}}}$, onde $\bar{x}$ e $\bar{y}$ são as médias das variáveis $x$ e $y$, respectivamente [14].
} 
0,97), ao passo que, para a imagem do ruído gaussiano, essa correlação é baixíssima $(\rho=0,002)$.

A existência de redundância indica que armazenar uma imagem como uma matriz em que cada elemento é a intensidade do pixel correspondente é ineficiente porque muitos pixels serão equivalentes [15]. Dependendo da base de representação (pixels, domínio de Fourier, coordenadas transformadas SVD), imagens podem ter aproximações mais compactas [1].

Figura 1: (a) Exemplo de imagem natural, (b) imagem gerada por ruído gaussiano, (c) gráfico de dispersão para pixels vizinhos da imagem mostrada em (a) e (d) gráfico de dispersão para pixels vizinhos da imagem do ruído gaussiano. As linhas tracejadas mostram as retas de correlação de Pearson igual à 1 .

(a)

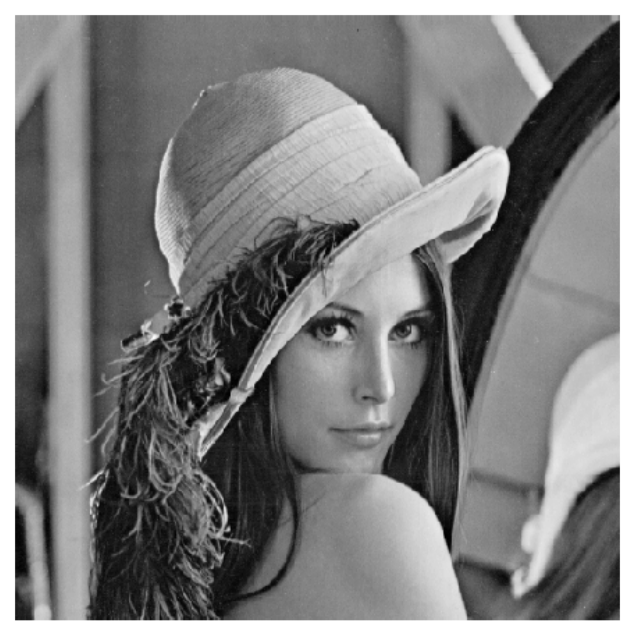

(c)

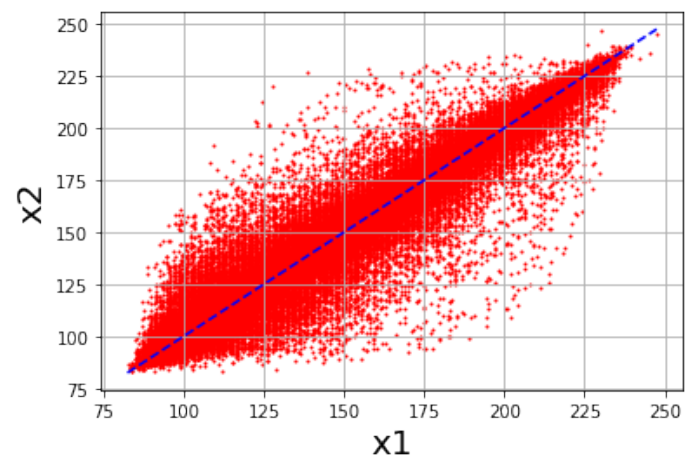

(b)

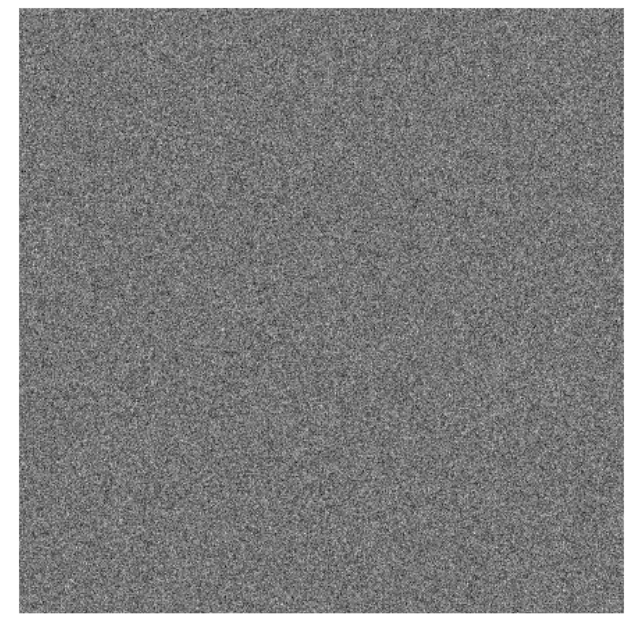

(d)

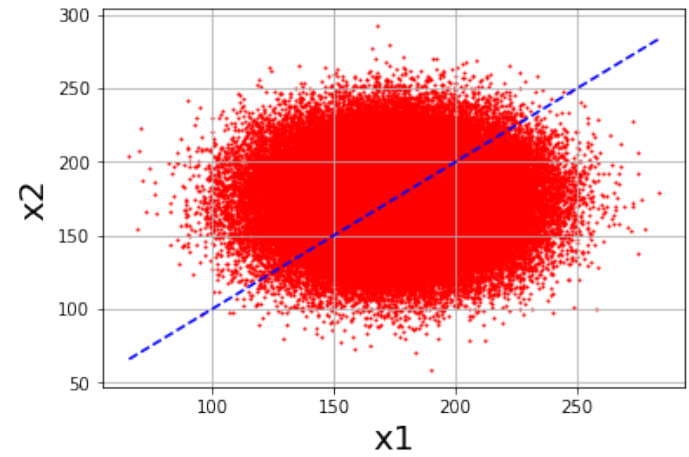

Fonte: (a) https://homepages.cae.wisc.edu/ ece533/images/lena.bmp, (b), (c) e (d) Autoral

Considere a ilustração do vírus Coronavírus (COVID-19), mostrada na Figura 2, que tem 2250 x 4000 pixels, ou seja, 9 milhões de amostras (9 megapixels). Calculando-se a decomposição SVD dessa imagem, obtemos $N=2.250$ valores sin- 
gulares $\sigma_{k}$. A Figura 3 mostra esses $k$ valores singulares. Observa-se que os valores singulares decaem rapidamente com o aumento de $k$, ou seja, a maior parte da informação da imagem está associada a um número de valores singulares muito menor do que 2.250 .

Figura 2: Ilustração criada pelo Center for Disease Control and Prevention (CDC) mostrando a morfologia do coronavirus.

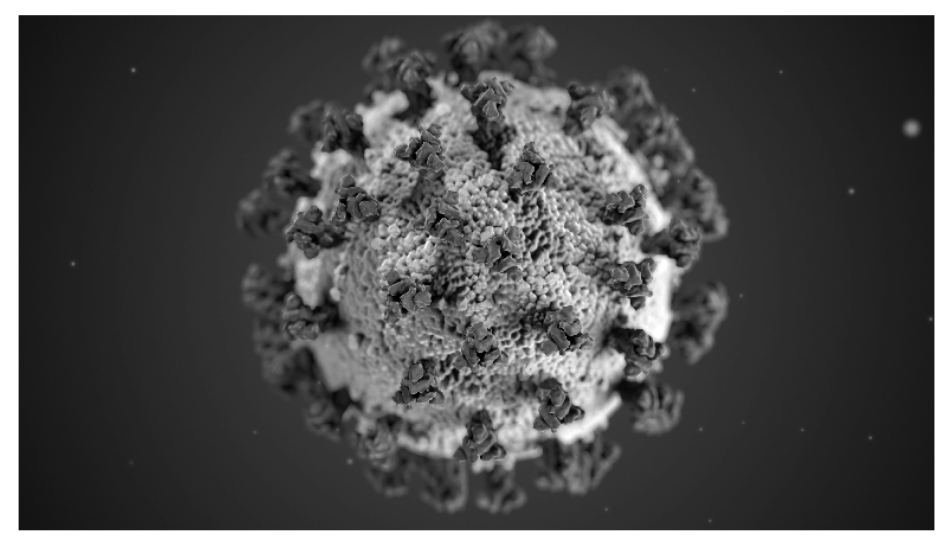

Fonte: Public Health Image Library. https://phil.cdc.gov/Details.aspx?pid=23311.

Figura 3: Valores singulares $\sigma_{k}$.

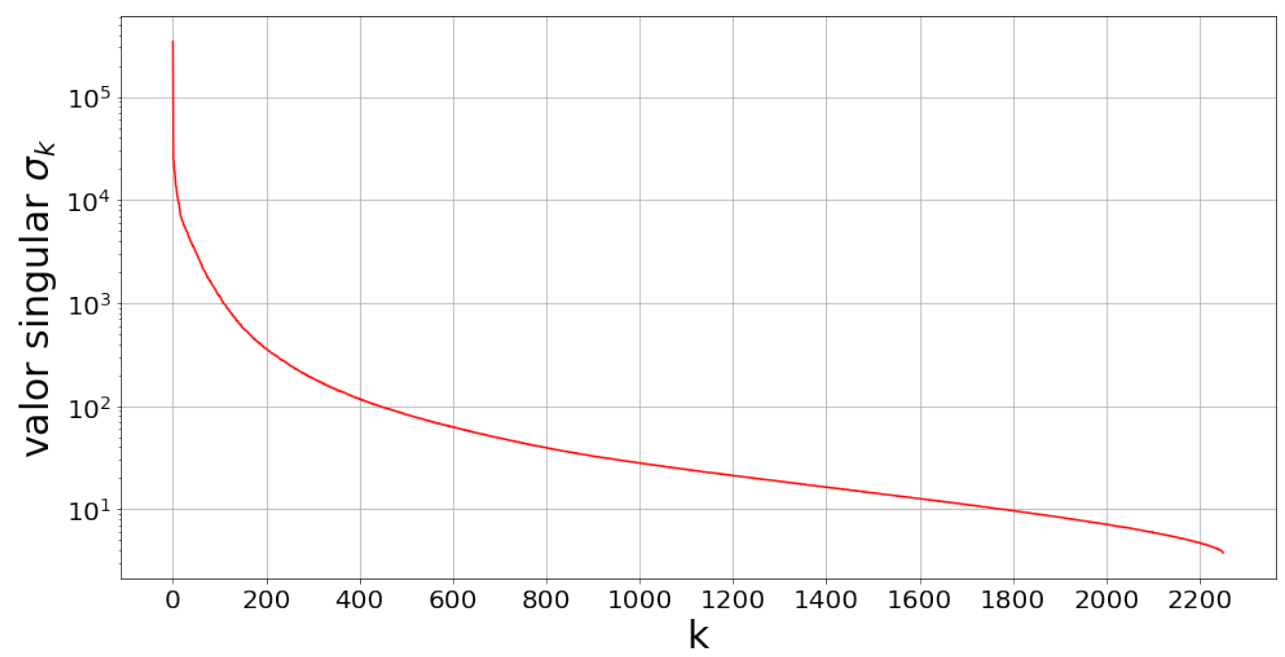

Fonte: Autoral.

Tomando-se os valores acumulados da soma dos $k$ primeiros valores singulares e normalizando pela soma total, podemos medir o percentual de informação que está contido nos primeiros $k$ valores singulares, ou seja, 


$$
\text { Percentual de Informação }=\frac{\sum_{i=1}^{k} \sigma_{i}}{\sum_{i=1}^{N} \sigma_{i}} \text {. }
$$

Figura 4: Valores singulares $\sigma_{k}$ acumulados.

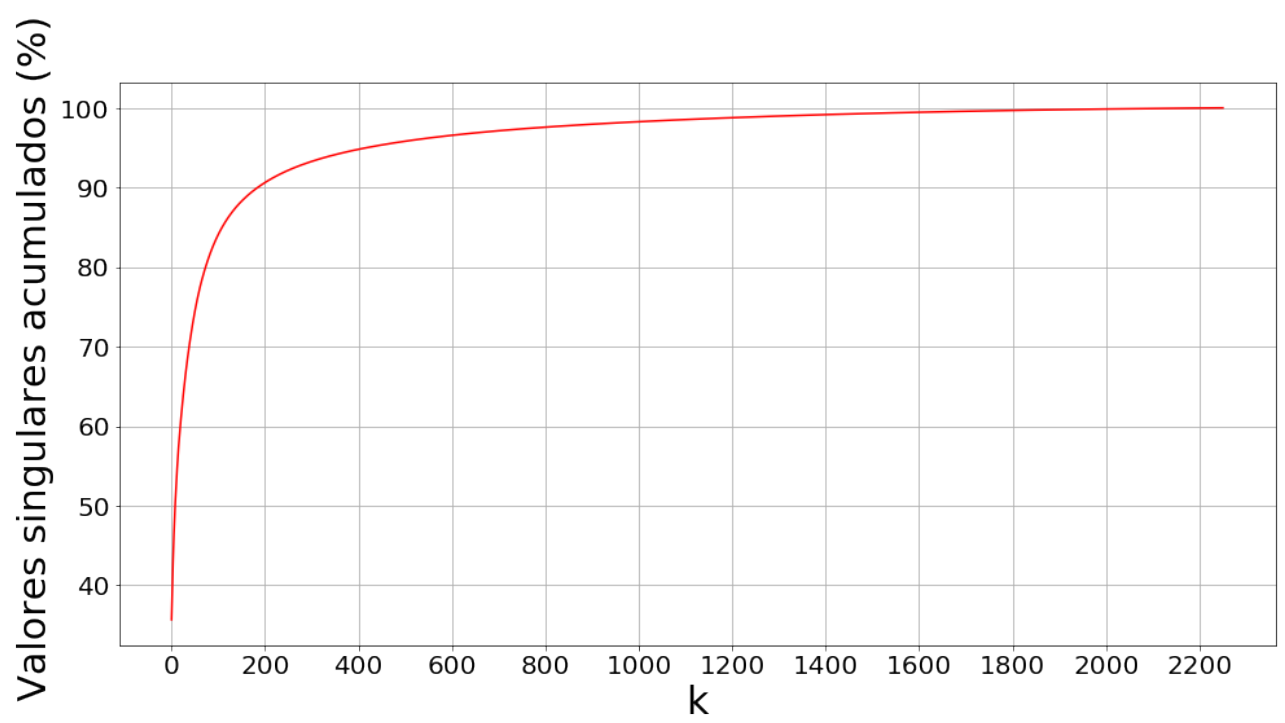

Fonte: Autoral.

A Figura 4 mostra esse percentual de informação contido nos primeiros $k$ valores singulares. A partir desses resultados, observa-se claramente a possibilidade de representar a imagem de uma forma comprimida usando apenas a quantidade de informação mais relevante. A matriz $X$, seguindo a Eq. (2.4), pode ser escrita na forma:

$$
X=\sum_{i=1}^{N} \sigma_{i} \mathbf{u}_{\mathbf{i}} \mathbf{v}_{\mathbf{i}}^{\mathbf{T}} .
$$

Considerando os $r$ primeiros valores singulares que mais contribuem para a imagem final, podemos aproximar a matriz $X$ pela matriz $\tilde{X}$, Eq. (4.9), e assim

$$
X \approx \tilde{X}=\sum_{i=1}^{r} \sigma_{i} \mathbf{u}_{\mathbf{i}} \mathbf{v}_{\mathbf{i}}^{\mathbf{T}} .
$$

Uma vez que o algoritmo para cálculo da decomposição SVD utiliza o método das potências, a velocidade de convergência depende da razão entre autovalores consecutivos $\frac{\left|\lambda_{i+1}\right|}{\left|\lambda_{i}\right|}$, ou analogamente, dos valores singulares consecutivos $\frac{\left|\sigma_{i+1}\right|}{\left|\sigma_{i}\right|}$. Portanto, a partir do gráfico mostrado na Figura 3, espera-se que a convergência seja mais rápida para os primeiros valores singulares, devido a maior diferença entre os valo- 
res singulares consecutivos, e se torne cada vez mais lenta para os valores singulares seguintes. A Figura 5 mostra o histograma do número de iterações necessárias para o cálculo dos primeiros 100 valores singulares. O número médio de iterações para essa faixa de valores singulares foi de 129, com $12 \%$ dos valores singulares necessitando do número máximo de iterações utilizado nos experimentos. No entanto, para os valores singulares restantes, a média foi de 197 iterações.

Figura 5: Histograma do número de iterações necessárias para o cálculo dos primeiros 100 valores singulares.

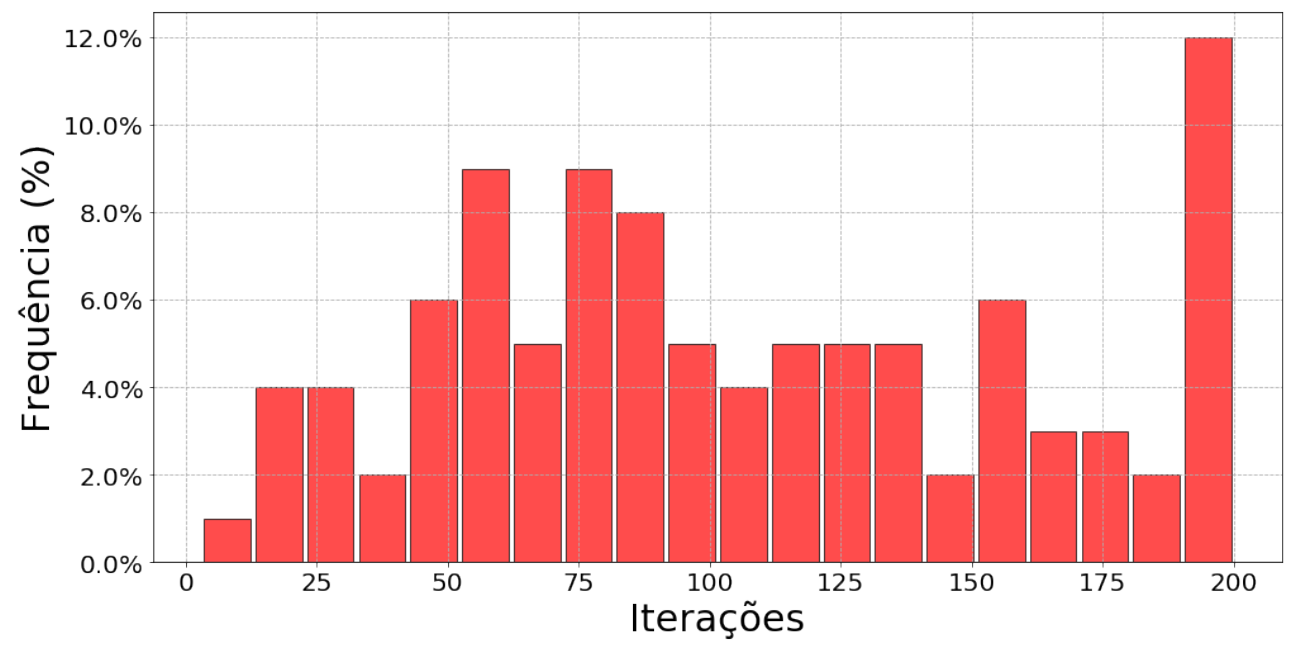

Fonte: Autoral.

A qualidade da imagem aproximada pode ser medida através do erro quadrático médio, em inglês Mean Square Error (MSE), definido como o quadrado da diferença entre os valores dos pixels da imagem original e da imagem aproximada ou comprimida dividido pelas dimensões da imagem dada por,

$$
M S E=\frac{\sum_{i=1}^{n} \sum_{j=1}^{m}\left(X_{i, j}-\tilde{X}_{i, j}\right)^{2}}{n m} .
$$

A razão de compressão $R$ para uma imagem comprimida é dada pela equação (4.11).

$$
R=100 \times \frac{r(n+m+1)}{n m}
$$

As Figuras 6-8 mostram as imagens aproximadas considerando os 10, 50 e 200 primeiros valores singulares. Observa-se a melhora na definição da imagem com o aumento do número de valores singulares usados na aproximação. No entanto, para r=200, já temos 90\% da informação contida na imagem original, sendo necessário 
Figura 6: Imagem construída com 10 valores singulares.

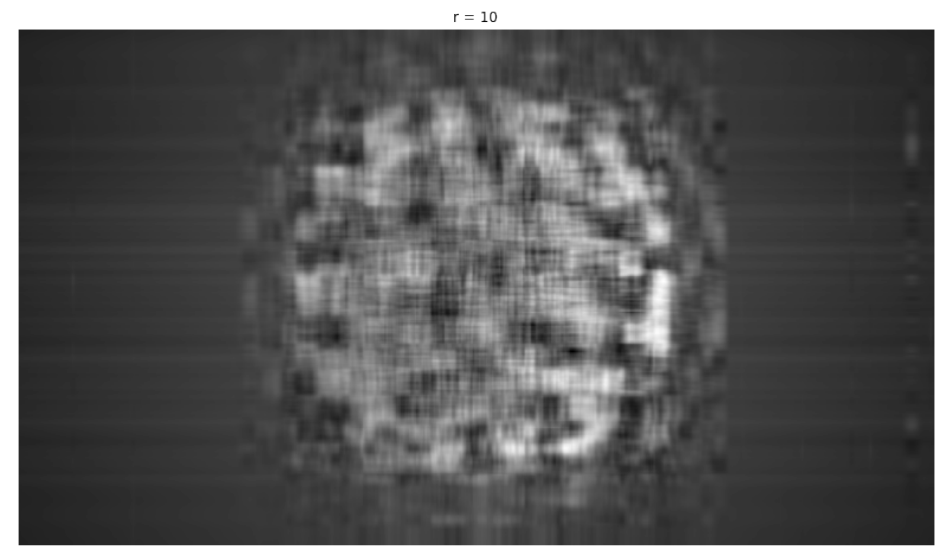

Fonte: Autoral

Figura 7: Imagem construída com 50 valores singulares.

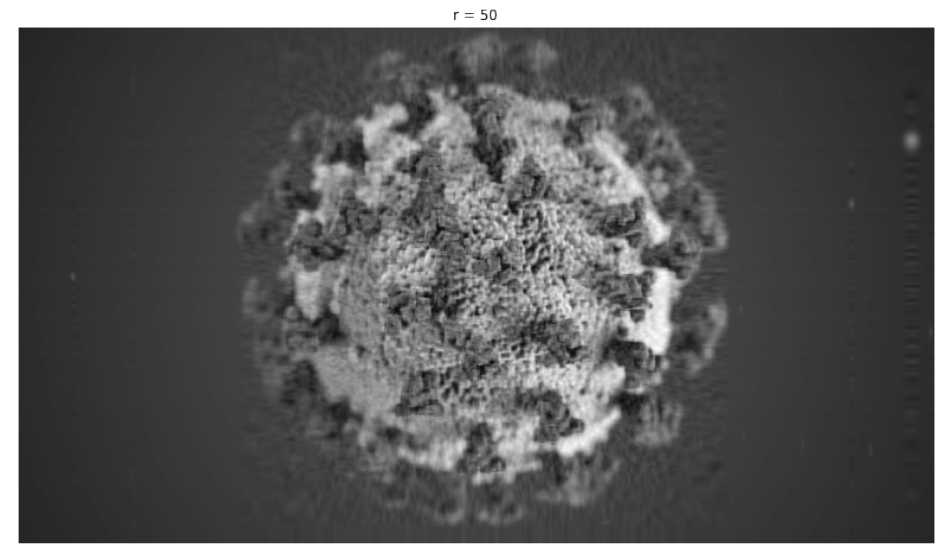

Fonte: Autoral

Figura 8: Imagem construída com 200 valores singulares.

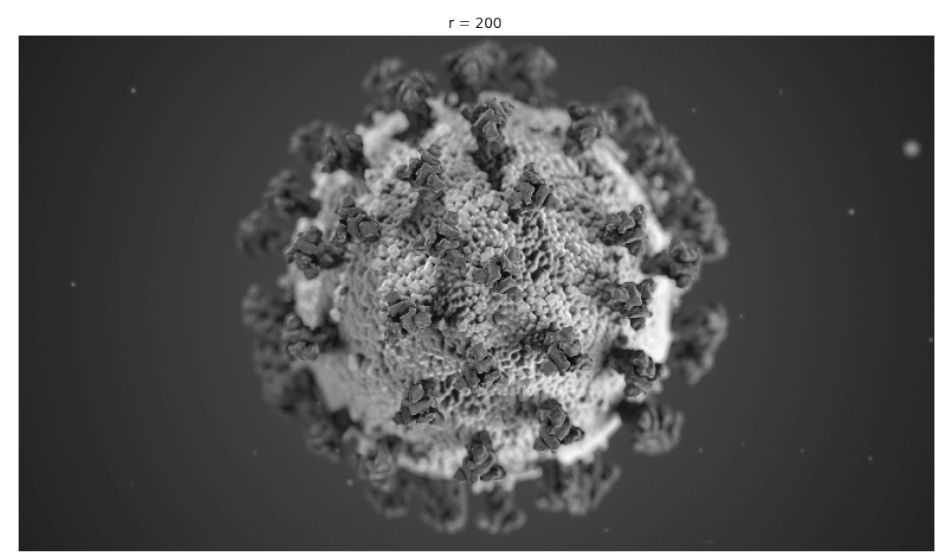

Fonte: Autoral 
armazenar 200 amostras para os valores singulares, $2250 \times 200$ amostras para os vetores $\mathbf{u}_{\mathbf{i}}$ e 200x4000 para os vetores $\mathbf{v}_{\mathbf{i}}^{\mathbf{T}}$, ou seja, 1.250.200 amostras. Isto representa apenas cerca de $14 \%$ das amostras originais.

A Tabela 1 mostra os valores do erro quadrático médio e o percentual de compressão para diferentes valores de $\mathrm{r}$ (número de valores singulares) variando de 10 até 1000. Estes valores comprovam numericamente o resultado já observado visualmente nas Figuras 6-8, ou seja, que os maiores valores singulares já possuem uma grande quantidade de informação da imagem permitindo uma boa visualização e assim possibilitando uma compressão nos dados permitindo uma redução no armazenamento.

Tabela 1: Erro quadrático médio (MSE) e Percentual de compressão (R) para diferentes valores de $\mathrm{r}$.

\begin{tabular}{ccc}
\hline$r$ & MSE & $\mathbf{R ( \% )}$ \\
\hline 10 & 28,05 & 3,47 \\
50 & 6,37 & 6,95 \\
200 & 1,29 & 13,89 \\
500 & 0,17 & 34,72 \\
1000 & 0,03 & 63,21 \\
\hline \multicolumn{3}{c}{ Fonte: Autoral }
\end{tabular}

\subsection{Autenticação de Imagens}

Outro exemplo interessante de aplicação da decomposição SVD é a autenticação de imagens. Uma alternativa para oferecer segurança aos documentos digitais é o uso de assinaturas digitais. Esse tipo de assinatura é caracterizada como marca d'água digital. O uso de marca d'água digital pode dificultar a falsificação e distribuição ilegal de conteúdos digitais. Uma boa marca d'água deve ser invisível ao sistema visual humano e interferir o mínimo possível no conteúdo digital da imagem hospedeira [16].

A autenticação de imagens utilizando da marca d'água digital pode ser dividida em três etapas: inserção, extração e verificação. Aqui apresentamos a metodologia baseada em [6] para realizar estas operações.

O processo de inserção da marca d'água é realizado tomando-se a decomposição SVD da imagem hospedeira ( matriz H, Eq. (4.12)), e da imagem utilizada como marca d'água (matriz W, Eq. (4.13)). Em seguida, modifica-se os valores singulares da imagem hospedeira de acordo com a equação (4.14) e se reconstrói a imagem autenticada através da equação (4.15). Os parâmetros $K$ e $M$ representam os números 
de valores singulares da marca d'água e da imagem hospedeira, respectivamente. De acordo com a equação (4.14), apenas os $(M-K)$ últimos valores singulares da imagem hospedeira, ou seja, aqueles menos representativos, serão modificados no processo de autenticação. O parâmetro $\alpha$ é um fator de escala que pode aumentar ou reduzir a fidelidade da imagem autenticada.

$$
\begin{gathered}
H=U_{H} S_{H} V_{H}^{T} . \\
W=U_{W} S_{W} V_{W}^{T} . \\
S_{m}=\left\{\begin{array}{cc}
S_{H}(i)+\alpha * \log \left(S_{W}(q)\right), & \text { se } M-K<i<M, 1 \leq q \leq K \\
S_{H}(i), & \text { se caso contrário. }
\end{array}\right. \\
A=U_{m} S_{m} V_{m}^{T} .
\end{gathered}
$$

Os processos de verificação e extração podem ser realizados aplicando as operações inversas, ou seja, toma-se a decomposição SVD da imagem autenticada (Eq. 4.16) e da imagem hospedeira, Eq. (4.12). Em seguida, calcula-se os valores singulares da marca d'água a ser extraída da imagem autenticada através da Eq. (4.17) e se reconstrói a marca d'água através da Eq. (4.18). Observe que o processo necessita do conhecimento prévio tanto da imagem hospedeira quanto da marca d'água utilizada.

$$
\begin{gathered}
A=U_{a} S_{a} V_{a}^{T} \\
S_{w}^{\prime}=\exp \left(\left(S_{a}(i)-S_{h}(i)\right) / \alpha\right), \quad \operatorname{para} M-K<i<M \\
W^{\prime}=U_{w} S_{w}^{\prime} V_{w}^{T}
\end{gathered}
$$

As Figuras 9 e 10 mostram o resultado de um processo completo de autenticação e verificação de uma imagem. A imagem mostrada na Figura 9(a) foi autenticada com a marca d'água mostrada na Figura 10(a). A imagem autenticada apresenta um erro quadrático médio de apenas 0,018 e a marca d'água é imperceptível. A marca d'água extraída é claramente legível e apresentou correlação de 0,78 com a marca d'água inserida. Aplicando-se o processo de extração e verificação à imagem 
original, ou seja, sem a marca d'água inserida, a marca d'água extraída apresenta-se ruidosa e pouco legível (Figura 10(c)), além de apresentar correlação de apenas 0,24 com a marca d'água original.

Portanto, apesar de simplificada, a utilização dessa metodologia se mostrou eficiente em inserir uma marca d'água imperceptível bem como de detectar a autenticidade de uma imagem previamente marcada e diferenciar uma imagem não marcada.

Figura 9: (a) Imagem original e (b) Imagem autenticada com a marca d'água mostrada na Figura 10(a).

(a)

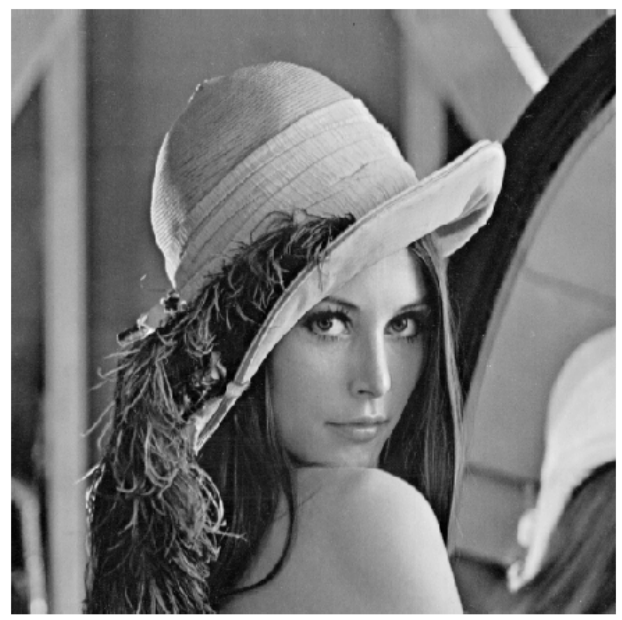

(b)

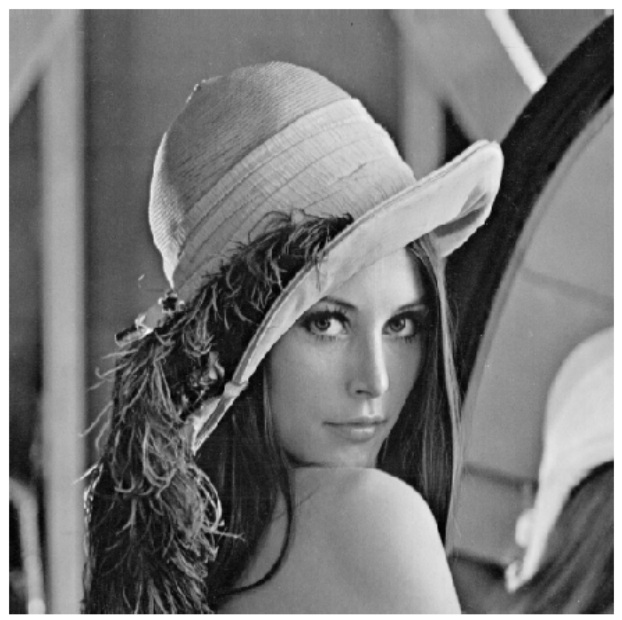

Fonte: (a) https://homepages.cae.wisc.edu/ ece533/images/lena.bmp e (b) Autoral.

Figura 10: Marcas d'água: (a) original, (b) extraída de imagem autenticada e (c) extraída de imagem não autenticada.

(a)

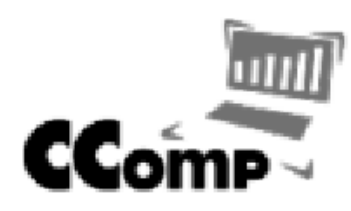

(b)

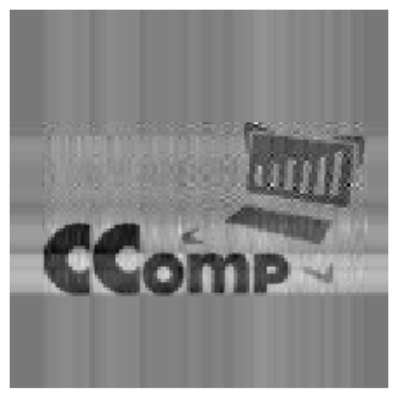

(c)

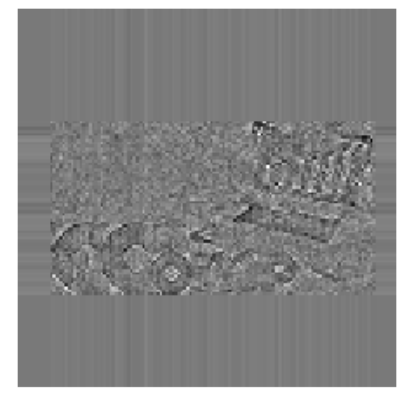

Fonte: (a) https://ccomp.ime.uerj.br/, (b) e (c) Autoral. 


\section{Conclusão}

A decomposição em valores singulares tem sido cada vez mais utilizada nas mais variadas áreas devido a sua existência garantida e versatilidade. Um algoritmo baseado no método das potências para obtenção dos valores singulares utilizados na decomposição SVD foi apresentado com base na exposição teórica do tema e foi posteriormente utilizado em duas aplicações na área de processamento de imagens: compressão e autenticação de imagens.

$\mathrm{Na}$ primeira, demonstrou-se a possibilidade de uso da técnica em compressão de imagens naturais levando a uma redução considerável do espaço utilizado para armazenamento com uma perda de qualidade controlada. Na segunda, um processo simples de autenticação e verificação de uma imagem através da inserção de uma marca d'água visualmente imperceptível foi reproduzido.

Os resultados apresentados mostram apenas uma pequena parcela dentre diversas possibilidades de aplicação prática da decomposição SVD, sobretudo na área de processamento de imagens. O propósito deste trabalho foi apresentar a utilidade, através da aplicação em exemplos reais, de conceitos teóricos matemáticos como autovalores, autovetores e valores singulares que normalmente são estudados nos

cursos de Álgebra Linear e o método das potências em Métodos Numéricos. Esperase que com este tipo de abordagem, este trabalho seja uma fonte de motivação para o entendimento destes conceitos básicos, bem como, um estímulo em trabalhos futuros, para o desenvolvimento de metodologias e algoritmos mais eficientes do que os aqui apresentados.

\section{Referências}

[1] BRUNTON, S. L.; KUTZ, J. N.: Data-Driven Science and Engineering: Machine Learning, Dynamical Systems and Control. Cambridge University Press, 2019.

[2] COGUBuM, M. C. T. H.: Transmissão de Imagens Utilizando SVD. Trabalho de Conclusão de Curso (Licenciatura em Matemática) - Departamento De Física/Química/Matemática, Universidade Federal de São Carlos - Campus Sorocaba, Sorocaba, 2013.

[3] GOLUB, G. H.; VAN LOAN, C. F.: Matrix Computations. 3 ed., Johns Hopkins University Press, 1996. 
[4] STEWART, G. W.: On the early history of the Singular Value Decomposition. SIAM REVIEW, v. 35, nº 4, p. 551-566, 1993.

[5] ERICHSON, N. B.; BRUNTON, S. L.; KUTZ, J. N.: Compressed Singular Value Decomposition for Image and Video Processing. In: IEEE International Conference on Computer Vision Workshops (ICCVW), Venice, p. 1880-1888, 2017.

[6] SADEK, R. A.: SVD Based Image Processing Applications: State of The Art, Contributions and Research Challenges. International Journal of Advanced Computer Science and Applications, v. 3, n 7, p. 26-34, 2012.

[7] PORSAni, M. J.; ARTOLA, F. A. V.; SilvA, M. G. da; MElO, P. E.M. de: Filtragem SVD aplicada à melhoria do rastreamento de horizontes sísmicos. Revista Brasileira de Geofísica, v. 28, n³ 3, p. 473-480, 2010.

[8] ANTON, H.; RORRES, C.: Álgebra Linear com Aplicações. Bookman, 2012.

[9] PRADHAn, T.; ROUTRAY, A.; KABI, B.: Comparative Evaluation of Symmetric SVD Algorithms for Real-Time Face and Eye Tracking. Matrix Information Geometry. 1. ed., Springer Berlin Heidelberg, 2013.

[10] PLASSMAN, G. E.: A survey of singular value decomposition methods and performance comparison of some available serial codes. NASA Technical Report, 2005.

[11] MELO, M. P. de: Ordenação das páginas do Google - "Page Rank". Dissertação (Mestrado em Estatística) - Instituto de Matemática e Estatística da Universidade de São Paulo, São Paulo, 2009.

[12] FRAnCO, N. B.: Cálculo Numérico. Pearson Prentice Hall, 2006.

[13] JAYARAMAN, J. T.; KARTHIKEYAN, N. R., PAVAN, T.; ANDREAS, S.: Image Understanding Using Sparse Representations. Synthesis Lectures on Image, Video, and Multimedia Processing. Morgan \& Claypool Publishers, 2014.

[14] HEUMANN, C.; SCHOMAKER, M.; SHALABH: Introduction to Statistics and Data Analysis: With Exercises, Solutions and Applications in R. Springer, 2016. 
[15] SCHUlZ, A.; SILVA, E. A. B. da; VELHO, L.: Compressive Sensing. 1. ed., Rio de Janeiro, Associação Instituto Nacional de Matemática Pura e Aplicada - IMPA, 2009.

[16] SILVA, J. F.; DUARTE, M. A. Q.; ALVARADO, F. V.: Inserção e Extração de Marca d'água em Imagens Digitais no Domínio Wavelet. Proceeding Series of the Brazilian Society of Applied and Computational Mathematics, v. 1, n 1, p. 1-6, 2013. 